\title{
Anticardiolipin antibody levels in diabetic subjects with and without coronary artery disease
}

\author{
T.J. Hendra ${ }^{1}$, E. Baguley², E.N. Harris ${ }^{2}$, M.H. Khamashta ${ }^{2}$, R.C. Trembath ${ }^{3}$, \\ G.R.V. Hughes ${ }^{2}$ and J.S. Yudkin ${ }^{1}$

\begin{abstract}
${ }^{1}$ The Academic Unit of Diabetes and Endocrinology of the Department of Medicine, University College and Middlesex School of Medicine, Archway Wing, Whittington Hospital, Highgate Hill, N19 5NF, ${ }^{2}$ The Lupus Arthritis Research Unit, The Rayne Institute, St Thomas Hospital, London SE1 7EH and ${ }^{3}$ The Medical Professorial Unit, St Bartholomew's Hospital, London ECIA 7BE, UK.
\end{abstract}

\begin{abstract}
Summary: Moderate ( $>20$ units) and high ( $>80$ units) IgG anticardiolipin antibody (aCL) titres are strongly predictive for recurrent thrombosis and early myocardial infarction in non-diabetic subjects. We have tested the hypothesis that the excess risk of myocardial infarction in diabetic subjects relates to the presence of aCL by measuring the frequency and titre of aCL in two groups of diabetic subjects and in 2500 healthy controls. One non-diabetic subject (0.04\%) had low (5-20 units) IgG aCL titres. Seven out of 126 diabetics without cardiovascular disease (5.6\%) and 9 out of 79 diabetics who were either myocardial infarction survivors or who had angiographically-proven coronary artery disease $(11.4 \%)$ had low aCL titres $(P<0.01$ for comparison of either diabetic group with controls, and $P<0.1$ for comparison between diabetic groups). One subject in each diabetic group, but no non-diabetics, had moderate IgM aCL titres. No subjects had high aCL titres. Diabetics have an increased frequency of low aCL titres which may relate to macrovascular disease. Macrovascular disease in diabetics is not associated with moderate or high aCL titres.
\end{abstract}

\section{Introduction}

The incidence of, and mortality from, coronary artery disease are increased in both insulin-dependent ${ }^{1}$ and non insulin-dependent ${ }^{2}$ diabetic subjects. The cause of this increased risk is unknown although it is possible that abnormalities of haemostasis described in diabetes $^{3}$ may be of aetiological significance.

Anticardiolipin antibodies $(\mathrm{aCL})$ have been found in non-diabetic subjects with autoimmune disease in association with severe peripheral vascular disease. ${ }^{4}$ The presence of high (greater than 80 units) and moderate (20-80 units) levels of $\mathrm{IgG}$ aCL in subjects with autoimmune disease has been shown to be strongly predictive for thrombosis, recurrent fetal loss and thrombocytopenia. ${ }^{5}$ In young subjects recovering from acute myocardial infarction, but without apparent autoimmune disease, the presence of aCL is associated with a high risk of recurrent vascular events. ${ }^{6}$

In order to test the hypothesis that the excess risk of coronary heart disease in diabetic subjects is related to

Correspondence: T.J. Hendra, B.Sc., M.R.C.P. (UK), Academic Unit of Diabetes and Endocrinology, Archway Wing, The Whittington Hospital, Highgate Hill, London N19 5NF, UK.

Accepted: 1 November 1988 an excess of aCL we have compared the frequency and titre of aCL in diabetics with and without coronary artery disease, and in a group of healthy controls.

\section{Subjects and methods}

The diabetic subjects without clinical evidence of coronary artery disease were selected at random from the diabetic outpatient clinic of the Whittington Hospital. The 126 patients were all well, with no illness other than diabetes mellitus. They all had a negative cardiovascular history for claudication or angina and had no previous history of vascular events. All had normal electrocardiograms and all peripheral pulses were palpable. None had a personal history or close family history of connective tissue disease. Thirty percent of $\mathrm{G}$ these subjects had microvascular complications of $N$ either diabetic nephropathy or retinopathy or both.

The 79 diabetics with coronary artery disease had 0 either been admitted to the Whittington or St Bar- 0 tholomew's Hospitals with an acute myocardial infarction confirmed according to WHO criteria $\stackrel{7}{\rightarrow}$ $(n=55)$ or had had severe coronary artery disease $T$ confirmed angiographically $(n=24)$. The diabetic $\frac{O}{\mathbb{D}}$ group comprised 19 insulin-dependent (mean age at

(C) The Fellowship of Postgraduate Medicine, 1989 
infarction $59 \pm 4$ (s.d.) years, mean duration of diabetes $22 \pm 5$ (s.d.) years) and 60 non-insulin dependent diabetics (age $62 \pm 6$ years, diabetes duration $8 \pm 9$ years). The majority of blood samples from this group were obtained at least 12 months after myocardial infarction. The 2500 healthy control subjects studied were drawn from blood bank donors (aged $18-65$ years).

Samples of sera from all subjects were stored at -20 or $-70^{\circ} \mathrm{C}$ and were then analysed for the presence of IgG and IgM anticardiolipin antibodies using a previously described ELISA technique. ${ }^{8}$

\section{Results}

The diabetic groups contained similar numbers of insulin-dependent patients but the patients with cardiovascular disease were older and had had diabetes for a shorter time than the disease-free group (Table I).

One healthy volunteer $(0.04 \%)$ had low IgG aCL titres (Table II). Seven (5.6\%) diabetics without vascular disease and $9(11.4 \%)$ diabetics with vascular disease had low IgG or IgM aCL titres $(P<0.01$ for comparison of either diabetic group with controls, and $P<0.1$ for comparison between diabetic groups). One subject in each diabetic group, but no nondiabetics, had moderate aCL titres. No subject in any group had high aCL levels (greater than 80 units). aCL titres were unrelated to diabetic treatment or to the presence of microvascular disease.

The diabetic patient in the vascular disease group with a moderate IgM aCL titre was a thin man who was a non-smoker and had had non-insulin dependent diabetes for 10 years prior to myocardial infarction at the age of 63 years. He had no islet cell, thyroid or parietal cell autoantibodies and he had had both legs amputated for peripheral vascular disease at 61 and 64 years of age.

The diabetic subject without vascular disease who had a moderate IgM aCL titre was a 71 year old man treated with tolbutamide $1 \mathrm{~g}$ daily who was normotensive and who had had diabetes for 3 years.

Table I Characteristics of diabetic subjects studied

\begin{tabular}{lccl}
\hline & Diabetics without & Diabetics with & \\
& $C A D$ & $C A D$ & \\
& $n=126$ & $n=79$ & Comparison \\
\hline Age (years) & $55.8 \pm 10.1 \dagger$ & $61.3 \pm 7.5$ & $P<0.001$ \\
Diabetes duration & $8.7 \pm 6.4 \dagger$ & $5.7 \pm 8.0$ & $P<0.01$ \\
Male/female ratio & $72 / 54$ & $40 / 39$ & $P>0.3$ \\
Number with IDDM & 21 & 19 & $P>0.2$ \\
\hline
\end{tabular}

CAD: coronary artery disease; IDDM: insulin-dependent diabetic subject; †mean \pm s.d.

Table II Anticardiolipin antibody positive subjects

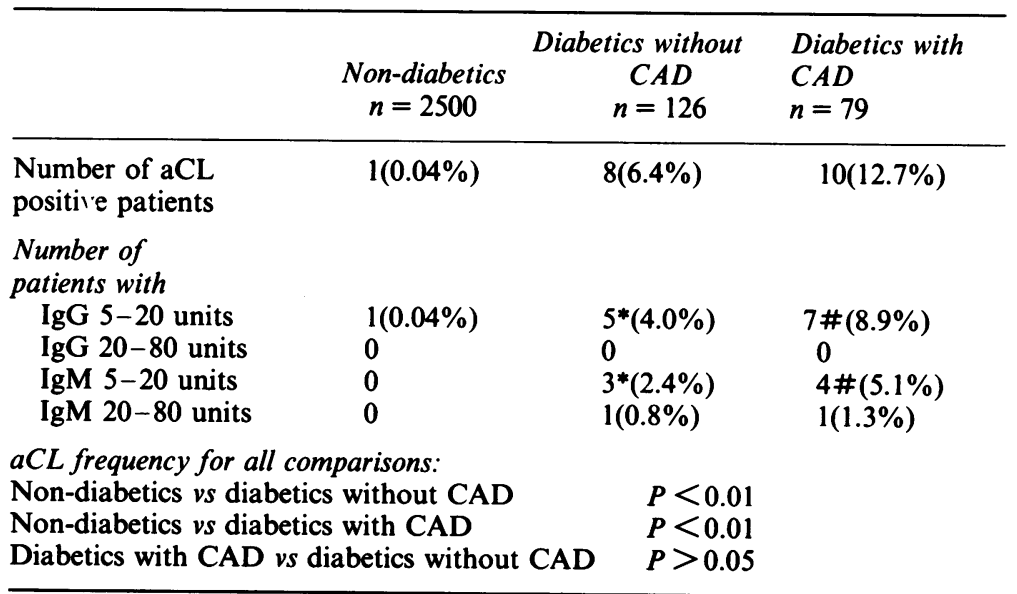

*Includes 1 patient with both IgG and IgM aCL 5-20 units; \# includes 2 patients with both IgG and IgM aCL 5-20 units; CAD: coronary artery disease. 


\section{Discussion}

In non-diabetic subjects the association between $\mathrm{aCL}$ and either early myocardial infarction or recurrent thrombosis may suggest that a causative role exists for these antibodies directed against negatively-charged phospholipids. Various mechanisms of action of $\mathrm{aCL}$ have been proposed. The presence of purified IgG with lupus anticoagulant activity may cause a reduction in prostacyclin release from rat aortic rings ${ }^{9}$ and cultured bovine ${ }^{9}$ and human ${ }^{10}$ endothelial cells. It has also been suggested that aCL may cause thrombocytopenia by binding to platelet membrane phospholipids thereby leading to platelet destruction. " However, a recent report has found no effect of $\mathrm{aCL}$ serum on human endothelial or platelet thromboxane $\mathrm{A}_{2}$ production. ${ }^{12}$ In addition to an effect on platelets or through prostanoid production, aCL may also influence haemostasis by an interaction with endothelial thrombomodulin resulting in reduced synthesis of protein C. ${ }^{13}$

Although insulin-dependent diabetes is associated with the presence of islet-cell and insulin autoantibodies, there is no recognized association with $\mathrm{aCL}$. In this study diabetic subjects both with and without vascular disease had an increased frequency of low titre aCL compared to healthy subjects. Although low IgG aCL titres are not as strongly associated with thrombosis as moderate or high titres, they still have a predictive value of $58 \%$ for thrombosis in patients with autoimmune disease (compared to $>70 \%$ for moderate and high aCL titres). ${ }^{5}$ Morton et al. ${ }^{14}$ demonstrated that the presence of low IgG aCL titres in subjects undergoing coronary artery bypass grafts is associated with an increased rate of graft occlusion. They also found a prevalence of $15 \%$ of low titre aCL in myocardial infarction survivors, which is similar to

\section{References}

1. Borch-Johnsen, K. \& Kreiner, S. Proteinuria: value as predictor of cardiovascular mortality in insulin dependent diabetes mellitus. Br Med J 1987, 294: 1651-1654.

2. Garcia, M.J., McNamara, P.M., Gordon, T. \& Kannel, W.B. Morbidity and mortality in diabetics in the Framingham population. Diabetes 1974, 23: 105-111.

3. Fuller, J.H., Keen, H., Jarrett, R.J. et al. Haemostatic variables associated with diabetes and its complications. Br Med J 1979, 2: 964-966.

4. Asherson, R.A., Derksen, R.H.W.M., Harris, E.N. et al. Large vessel occlusion and gangrene in systemic lupus erythematosus and 'lupus-like' disease. A report of six cases. J Rheumatol 1986, 13: 740-747.

5. Harris, E.N., Chan, J.K.H., Asherson, R.A., Aber, V.R., Gharavi, A.E. \& Hughes, G.R.V. Thrombosis, recurrent fetal loss and thrombocytopenia. Predictive value of the anticardiolipin antibody test. Arch Intern Med 1986, 146: 2153-2156. our findings in diabetic subjects. However, they found a higher aCL frequency of $29 \%$ in non-infarct subjects with coronary artery disease awaiting surgery.

Low aCL titres may not be a consistent finding after myocardial infarction. In the study by Hamsten et al. ${ }^{6}$ $21 \%$ of young myocardial infarction survivors had aCL demonstrable on 2 out of 3 separate occasions over the 3 year post-infarction period. However, they found that the pattern of aCL positivity after infarction may change so that of 18 patients who were positive on at least one occasion in the 3 year post-infarction period, 6 patients who had been positive at either 3 or 12 months (or at both timepoints), were negative at 3 years.

Our finding of no difference in low aCL titres between the diabetic subjects with and without vascular disease may be type 2 error since the $\chi^{2}$ value is only marginally non-significant. The relevance of low aCL titres in our diabetic subjects free of vascular disease is unclear. Although it is possible that low aCL titres may contribute to the risk of myocardial infarction, longitudinal studies may be required to evaluate the relevance of $\mathrm{aCL}$ to vascular disease in subjects with diabetes. It is unlikely that $\mathrm{aCL}$ are secondary to the well documented abnormalities of platelet and endothelial function described in diabetes. ${ }^{3}$

Thus, in conclusion, subjects with diabetes have an increased frequency of low titre $\mathrm{aCL}$ which is no@ related to the presence of micro- or macro-vasculat disease.

\section{Acknowledgement}

The authors are grateful to Dr D.J. Galton (St Bartholomew's Hospital) for allowing patients admitted under his care to be studied.

6. Hamsten, A., Norberg, R., Bjorkholm, M., De Faire, U \& Holm, G. Antibodies to cardiolipin in young survivors of myocardial infarction: an association with recurren cardiovascular events. Lancet 1986, i: 113-116.

7. World Health Organisation. Regional Office for Europe Public Health in Europe 5. Annex 1. World Health Organisation, Copenhagen, 1976. (Myocardial Infarction community registers.)

8. Gharavi, A.E., Harris, E.N., Asherson, R.A. \& Hughes, G.R.V. Anticardiolipin antibodies:isotype distribution and phospholipid specificity. Ann Rheum Dis 1987, 46: $1-6$.

9. Carreras, L.O., Defreyn, G., Machin, S.J. et al. Arterial thrombosis, intrauterine death and 'lupus' anticoagulant. Detection of immunoglobulins interfering with prostacyclin formation. Lancet 1981, i: 224-246. 
10. Schorer, A.E. \& Watson, K.V. The 'lupus anticoagulant' induces functional changes in endothelial cells and platelets. Thromb Haemost 1987, 58: 232.

11. Harris, E.N., Asherson, R.A., Gharavi, A.E., Morgan, S.H., Derue, G. \& Hughes, G.R.V. Thrombocytopenia in SLE and related disorders: association with anticardiolipin antibody. Br J Haematol 1985, 59: 227-230.

12. Hasselaar, P., Derksen, R.H.W.M., Blokziji, L. \& De Groot, P.G. Thrombosis associated with antiphospholipid antibodies cannot be explained by effects on endothelial and platelet prostenoid synthesis. Thromb Haemost 1988, 59: 80-85.
13. Cariou, R., Tobelem, G., Soria, C. \& Caen, J. Inhibition of protein $\mathrm{C}$ activation by endothelial cells in the presence of lupus anticoagulant. $N$ Engl J Med 1986, 314: $1193-1194$.

14. Morton, K.E., Krilis, S.A., Baron, D.W. et al. Coronary artery bypass graft failure - an autoimmune phenomenon. Lancet 1986, ii: 1353-1356. 